\title{
The Disjoint Path-Pair Matrix Approach for Online Routing in Reliable WDM Networks
}

\author{
Marco Tacca, Paolo Monti, Andrea Fumagalli \\ Optical Networking Advanced Research (OpNeAR) Lab \\ Erik Jonsson School of Engineering and Computer Science \\ University of Texas At Dallas \\ Richardson, TX 75080 \\ Email:\{mtacca,pxm017500,andreaf $\} @$ utdallas.edu
}

\begin{abstract}
In reliable Wavelength Division Multiplexed (WDM) networks, the optimal choice of Routing and Wavelength Assignment (RWA) for the working and protection path-pair is often a complex problem to solve.

To cope with such problem complexity, this paper presents the Disjoint Path-Pair Matrix (DPM) approach. With the DPM approach the RWA problem complexity - i.e., the size of the solution space - can be significantly reduced by limiting the number of candidate path-pairs considered in the optimization.

Simulation results are collected using the DPM approach to solve the online RWA problem in a network based on the Shared Path Protection switching scheme with Differentiated Reliability. When compared to the conventional $k$-shortest paths approach, the DPM approach requires up to one order of magnitude less candidate path-pairs. In addition, the DPM approach finds solutions with reduced hop length of both the working and protection paths by up to $3 \%$ and $14 \%$, respectively.
\end{abstract}

\section{INTRODUCTION}

One of the most valuable services offered by today's Wavelength Division Multiplexed (WDM) networks is the ability to provide high-bandwidth all-optical channels - also referred to as lightpaths [1]. Such lightpaths can be created between node pairs in the network to produce the desired logical connectivity. A WDM network can be made reliable by means of protection switching schemes implemented at the WDM layer [2]. A protection scheme requires allocation of spare resources to be used in the case of a network fault, e.g., with an end-to-end protection scheme, a connection demand is assigned both a working and a protection path.

WDM networks require that, for each lightpath to be created, both a route and a wavelength have to be assigned - the so called Routing and Wavelength Assignment (RWA) problem. Once transmitted at the source, the lightpath signal can reach the destination without requiring either electronic processing, or wavelength conversion. When traffic demands dynamically enter and leave the network, the problem is referred to as the online RWA problem. The objective of the online RWA problem is to reserve available network wavelengths to the incoming traffic demands in a way that the overall blocking probability is minimized - a demand is

This research was supported in part by NSF Grants No. ANI-0082085 and ECS-0225528, CPqD, and the Italian Ministry of University (MIUR) (contract no. RBNE01KNFP) blocked when it cannot be created due to the lack of available wavelengths.

Finding the optimum solution for the RWA problem is a challenging combinatorial problem, whose complexity i.e., the size of the solution space - grows with both the network size and the number of connections. Various proposed approaches [3], [4], [5] model this problem as a variation of the multicommodity flow problem [6]. They solve it by carefully pruning the set of candidate paths that can be chosen to provide a sub-optimal solution [7]. These approaches are based on the intuitive reasoning that a relatively small number of candidate paths may lead to a satisfactory solution of the multicommodity flow problem from both complexity and performance standpoint. A well-known pruning technique is based on the $k$-shortest paths found on the given network topology [8].

It can be shown that for unprotected networks a relatively small value of $k$ may already produce results that are close to the optimum. On the contrary, when dealing with reliable networks the use of the $k$-shortest paths may require a much larger value of $k$. The reason is twofold. First, at least one route disjoint path-pair — one path for the working path and the other for the protection path - must be found for each source-destination pair. This is a necessary condition to have a feasible solution to the RWA problem in a reliable network. Second, a sufficient number of distinct path-pairs must be available for each source-destination pair. This latter condition is necessary to allow some degree of flexibility in choosing the best path-pair for any given demand. As shown in Section V the simplistic approach based on the single shortest disjoint path-pair [6], [9] may not yield satisfactory performance.

Unfortunately, a number of drawbacks may arise when large values for $k$ are required. First, the number of possible path-pairs grows with $k$, making the problem increasingly difficult to solve. Second, the average hop length of the $k$ shortest paths increases with $k$, possibly causing the end-toend connection quality to degrade below the desired level. Additionally, a practical constraint in reliable networks is that the hop difference between the working path and the protection path of the same demand should be minimal. This constraint avoids tangible quality of service degradation when traffic is forced to switch from the working to the protection path, and 
vice versa.

This paper proposes an alternative pruning technique to the $k$-shortest paths. The objective of the proposed pruning technique is manifold:

- to control and limit the number of route disjoint candidate path-pairs,

- to control and limit the number of hops of the working paths,

- to control and limit the number of hops of the protection paths,

- to control and limit the hop difference between the working and the protection paths.

All these objectives are accomplished while maintaining a solution performance that is comparable to the - less controllable - solution obtained with the $k$-shortest path pruning approach. As described in detail in Section III, a unique set of candidate path-pairs is chosen to form the Disjoint Path-Pair Matrix (DPM) for each source-destination pair. By limiting the number of candidate path-pairs in the DPM, the proposed approach greatly reduces the solution space, while maintaining the necessary flexibility to find an acceptable sub-optimal solution to the RWA problem in reliable WDM networks.

The advantages of the DPM approach are investigated by solving the online RWA problem in a network in which protection is provided by means of the Shared Path Protection (SPP) switching scheme with Differentiated Reliability (DiR) [10], [11]. The SPP-DiR scheme provides end-to-end protection as opposed to providing segmented backup protection [12], [11]. The SPP-DiR scheme is chosen here for two reasons. First, it is one of the most cost-effective protection schemes that provide the desired degree of reliability in WDM networks [13]. Second, the complexity of its RWA problem is higher than the complexity of many other protection schemes, including the conventional SPP. Thus, it represents a good candidate to test the validity of the proposed DPM approach.

\section{Drawbacks of the $k$-Shortest Paths ApproACH}

As already mentioned, most approaches available in the literature are based on a preselection of paths based on the well-known $k$-shortest paths algorithm [14], [15]. This approach has been successfully applied to the problem of routing working paths in WDM networks [16] without providing survivability. However, when protection is required, the approach based solely on preselecting the $k$-shortest paths may present a number of disadvantages, as illustrated by the following example. In Figure 1, when the source-destination

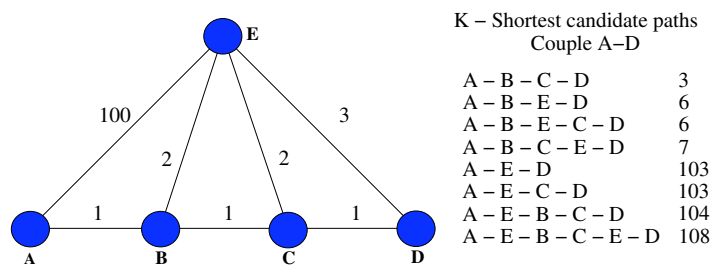

Fig. 1. Example pair A-D is considered, there are no route disjoint path-pairs among the first 4 shortest paths. If at least one route disjoint path-pair is required for protection purposes, a larger number of shortest paths has to be considered. While this example is artificially built to illustrate the issue, similar cases can be found in real world networks. It is evident that two major drawbacks exist with the $k$-shortest paths approach. First, the way to determine the minimum number of shortest paths found by a $k$-shortest path algorithm [8] that are required to find at least one route disjoint path pair is trial and error. In addition, if the number of candidate shortest paths is substantially large, the network designer has little control over both the length and the length difference between working and protection paths.

\section{Construction OF THE DPM}

One Disjoint Path-Pair Matrix is built for each sourcedestination pair. The DPM is computed beforehand and is then used to route all the incoming connection demands. The DPM approach is based on the observation that the space of possible solutions contains only route disjoint path pairs. The idea consists of preselecting a desired number of candidate working paths $\left(k_{1}\right)$ using the $k$-shortest paths approach for each sourcedestination pair. For each candidate working path a number of candidate disjoint protection paths $\left(k_{2}\right)$ is computed ${ }^{1}$. A matrix of candidate route disjoint path-pairs is thus available for each source-destination pair. Hence the name Disjoint Path-Pair Matrix (DPM). The matrix has dimensions $k_{1} \times k_{2}$. The row index $0 \leq i<k_{1}$ identifies the candidate working path. The column index $0 \leq j<k_{2}$ identifies the candidate protection path.

In general, the DPM approach can be applied to any protection scheme based on end-to-end rerouting, i.e., Shared Path Protection (SPP) scheme or Dedicated Path Protection (DPP) scheme [17]. For demonstration purposes, in this paper the DPM approach is applied to solving the Routing and Wavelength Assignment (RWA) problem of the Shared Path Protection (SPP) scheme with Differentiated Reliability (DiR).

\section{THE SPP SCHEME WITH DIR}

This section first provides a brief description of the Shared Path Protection scheme with Differentiated Reliability (SPPDiR). (For a more complete description of this scheme, the reader is referred to [10], [13].) The online RWA problem associated with the SPP-DiR scheme is then defined assuming dynamic traffic requests and finite network capacity. Finally, the Simulated Annealing algorithm based on the precomputed DPM is described.

According to the Differentiated Reliability concept, each connection is characterized by a maximum failure probability (or downtime ratio) that must be met by the protection scheme. The ultimate objective of the SPP-DiR design is to satisfy the reliability degree of the traffic demand, while optimizing the network performance.

${ }^{1}$ It might be possible, due to the topology layout, that particular node pairs have a number of candidate working paths smaller than $k_{1}$. The same observation applies to the number of candidate protection paths, $k_{2}$. 


\section{A. Network Model}

The RWA problem of the SPP-DiR scheme applied to WDM mesh networks is formulated next. It is assumed that a lightpath requires the same wavelength on every link of the path chosen to connect two nodes. The WDM network is modeled using a graph $G(\mathcal{N}, \mathcal{L})$, where $\mathcal{N}$ represents the set of network nodes and $\mathcal{L}$ is the set of network links. Each link in the network has $F$ fibers for each direction of propagation, and each fiber can support $W$ wavelengths. Wavelength conversion is not available in the network. However, working and protection paths provisioned to the same traffic demand may use distinct wavelengths. It is assumed that only single faults may occur in the network at once, i.e., the probability that two or more links are concurrently failed is assumed to be negligible. It is also assumed that a link fault disrupts all demands along both direction of propagation.

All the links in set $\mathcal{L}$ are characterized by three parameters: the number of available fibers in the link, the set of available wavelengths, and the value of the link conditional failure probability. Based on the single fault assumption made earlier, the conditional link failure probability is the conditional failure probability given that a single link fault has occurred in the network ${ }^{2}$. For example assuming a uniform distribution of faults among the links, the conditional link failure probability is $P_{f}(i, j)=\frac{1}{|\mathcal{L}|} \forall(i, j) \in \mathcal{L}$.

Connection demands dynamically arrive at network nodes and must be served as they are received. A demand consists of one lightpath that needs to be created between two nodes. A lightpath is a path of light between a node pair whose bandwidth equals the wavelength bandwidth.

Differentiated Reliability is achieved by assigning to each demand the minimum amount of resources needed to ensure the required level of reliability. The reliability degree is modeled by assigning each demand a Maximum Conditional Failure Probability $(M C F P)$. The value of $M C F P$ represents the maximum acceptable probability that, given a network link failure, the connection will not survive. The protection scheme must satisfy this value for each demand. While the conventional SPP protection scheme is able to provision each demand with resources to be $100 \%$ survivable against any single fault, i.e., the SPP scheme supports only $M C F P=0$, the SPP-DiR scheme yields a wider range of possible $M C F P$ values.

A demand $D$, associated with a non-stringent $M C F P$ value, does not need a protection path for every possible link failure scenario. The SPP-DiR scheme selects a set of links $U^{(D)}$ of the working path. If one of those links fails demand $D$ will not require resorting to the protection path. Notice that, with SPP-DiR two working paths having a common link along the working path, can also share protection wavelengths if at least one of the two working paths will not resort to the protection path in case of a failure of the common working

\footnotetext{
${ }^{2}$ Given the single fault assumption the link failure probability is the product between the conditional link failure probability and the probability of having a single fault.
}

link. Similarly, it is also possible to have a working path completely unprotected if its path failure probability satisfies the reliability requirement given by the connection $M C F P$. Intuitively, the SPP-DiR scheme achieves a better resource utilization when compared to the conventional SPP scheme, while provisioning to each connection enough resources to satisfy the reliability requirement.

\section{B. Solving the SPP-DiR RWA Problem}

A two-step Simulated Annealing (SA) algorithm based on DPM path preselection is used to solve the online RWA problem for SPP-DiR. The algorithm objective is to minimize the overall network blocking probability.

The DPM path pairs are used by a Simulated Annealing (SA) based algorithm to find the best candidate path-pair that can be used to provision resources to incoming demands. The SA algorithm used in this paper differs from the one presented in [10] due to the possibility of an additional move. The additional move consists of attempting to modify the routing of the working path. After finding the initial solution using a first fit approach, at each iteration not only the protection path but also the working path can be changed. This is accomplished by the SA based algorithm by randomly choosing one path in the first column of the DPM matrix.

\section{RESULTS}

The proposed DPM approach presented in Section III is tested using the European optical network topology (Figure 2(a)). This network comprises 19 nodes and 39 bidirectional links. It is assumed that each link accommodates 1 fiber for each direction of propagation and each fiber carries 32 wavelengths. The conditional link failure probability is obtained assuming a uniform distribution of faults among the links. Therefore, the single link conditional failure probability is $P_{f}(i, j)=\frac{1}{39}$.

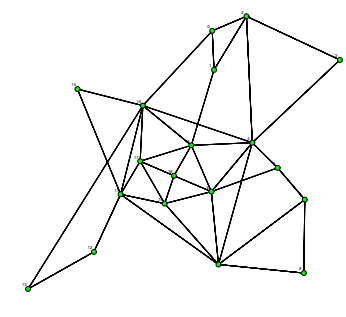

(a)

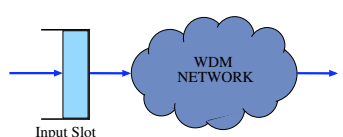

(b)
Fig. 2. Pan-European optical network (a) and input slot buffer (b).

Traffic demands are generated according to a Poisson distribution with arrival rate $\lambda$. Source and destination nodes are randomly chosen with a uniform distribution among all possible node pairs. Each demand requires one lightpath and is assigned a predetermined fixed reliability degree requirement of $M C F P=0.03$. With this value and in the network topology under consideration, each demand may be able 
TABLE I

PATH PRESELECTION STATISTICS

\begin{tabular}{|c|c|c|c|c|c|c|c|}
\hline Scheme & $k_{1}$ & $k_{2}$ & $N_{W}$ & $N_{P}$ & $N_{p p}$ & $H_{c_{w}}$ & $H_{c_{P}}$ \\
\hline LB & \multicolumn{2}{|c|}{$k=60$} & 60 & 15.868 & 952.1 & 5.008 & 4.646 \\
\hline DPM & 30 & 5 & 30 & 4.685 & 140.5 & 4.507 & 3.803 \\
\hline DPM & 20 & 10 & 20 & 9.289 & 185.8 & 4.157 & 4.438 \\
\hline DPM & 20 & 5 & 20 & 4.688 & 93.8 & 4.157 & 3.819 \\
\hline$\overline{D P M}$ & 10 & 10 & 10 & 9.338 & 93.4 & 3.604 & 4.459 \\
\hline DPM & 10 & 5 & 10 & 4.709 & 47.1 & 3.604 & 3.875 \\
\hline
\end{tabular}

to have up to one working link that is unprotected. Once established, a demand remains in the system for a time that is exponentially distributed with parameter $\frac{1}{\mu}=1$. It is assumed that the signaling latency in the network is negligible, and the correct network status information is available at all nodes.

To provide results that are not dependent upon any specific call admission control, all arriving demands are first stored in a virtual centralized buffer, as shown in Fig. 2(b). At most one demand can be stored in the buffer at once. A demand, that upon arrival cannot be established in the network due to lack of available resources, is stored in the buffer until it can be established. Demands that arrive while the buffer is busy are blocked and dropped.

The SA algorithm requires some parameters to be fine tuned in order to achieve good results in terms of both quality of the solution found and computational time required to find the solution. It was found that a number of iterations, $n$ rep $=100$, a starting temperature of $t_{0}=6$, a final temperature of $t_{f}=1$, and a cooling factor $a=0.9$ with geometrically decaying temperature, represent a good trade-off in terms of the above objectives. A number of simulations were run for each point to achieve a confidence interval value of $18 \%$ or better at $95 \%$ confidence level.

The proposed DPM path preselection is compared to a path pruning technique based on the $k$-shortest paths approach termed Linear Based (LB) path preselection. For LB, candidate path-pairs are computed as follows. For any possible node pair, only the first $k$ shortest loopless paths are considered. All the possible route-disjoint path-pairs that can be generated from the considered $k$ candidate paths are then used to create the LB matrix. The LB matrix is then used by the RWA algorithm described in Section IV-B.

Table I shows some statistics that are collected on the routedisjoint pair-paths obtained by both the DPM and LB pruning techniques. From left to right the table reports: the preselection scheme used, the value of $k_{1}$ and $k_{2}$ used for building the DPM matrix, the average number of candidate working paths per source-destination pair $\left(N_{W}\right)$, the average number of candidate protection paths associated with each working path $\left(N_{P}\right)$, the average number of candidate route disjoint path-pairs per source-destination pair $\left(N_{p p}\right)$, the average hop length of a candidate working path $\left(H_{c_{w}}\right)$, and the average hop length of a candidate protection path $\left(H_{c_{p}}\right)$. For the LB approach, $k=60$ shortest paths between each source-destination pair are used. For the DPM approach five configurations are considered: $k_{1}=30$ candidate working paths with $k_{2}=5$ protection paths, $k_{1}=20$ candidate working paths with $k_{2}=10$ and $k_{2}=5$ protection paths, and $k_{1}=10$ candidate working paths with $k_{2}=10$ and $k_{2}=5$ candidate protection paths.

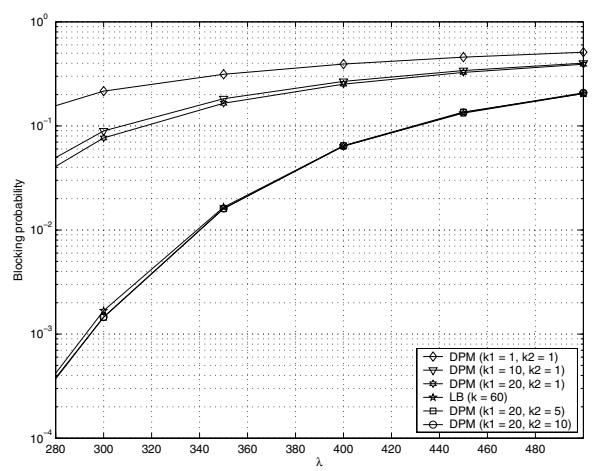

Fig. 3. Blocking probability

The values reported in Table I support the earlier claim that by using the DPM pruning technique the size of the solution space may be reduced when compared to the LB solution space. In some instances, i.e., when comparing LB with $k=60$ and DPM with $k_{1}=20$ and $k_{2}=5$, the DPM approach is able to reduce the solution space by one order of magnitude. Table I also shows that with the DPM pruning it is possible to better control the hop length of both the working and protection paths.

Figure 3 reports the overall network blocking probability. The curves show that the DPM based algorithm can better solve the RWA problem due to the reduced size of the solution space $^{3}$. The figure also shows the importance of having multiple candidates in obtaining satisfactory performances: when the values for $k_{1}$ and or $k_{2}$ are too small, the blocking is negatively and significantly affected.

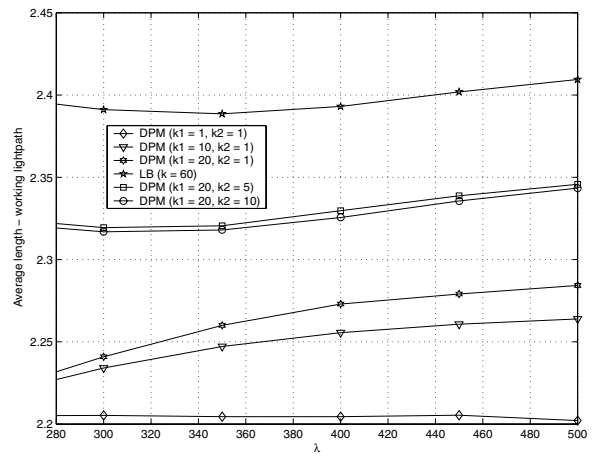

Fig. 4. Average length of the provisioned working path

Figures 4 and 5 report the average hop length of both the working path and the protection path. The plots reveal that the DPM based approach is effective in reducing the length of both the working and the protection paths under any traffic

\footnotetext{
${ }^{3}$ The comparison is performed using the same values of the SA parameters,
} therefore the running times of the two approaches are comparable. 
load. Figure 6 reports the average number of links in which sharing of protection resources is possible along the protection path.

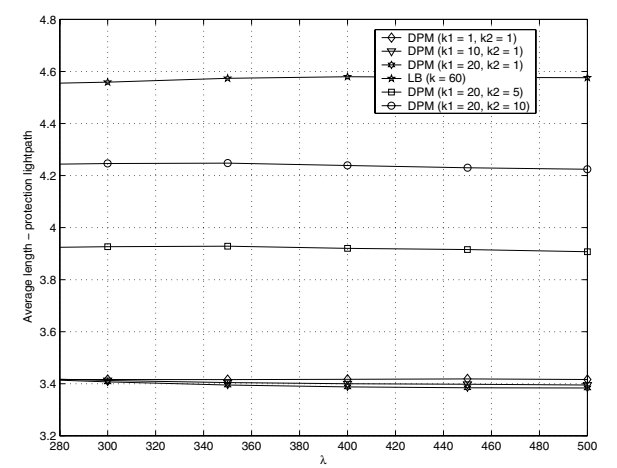

Fig. 5. Average number of the provisioned protection path

Table II reports the performance of the DPM approach in the five configurations described in table I. The table reports the overall network blocking probability $(B P)$, the average hop length of the chosen working path $\left(H_{W}\right)$, and the hop length of the chosen protection path $\left(H_{P}\right)$, as a function of $k_{1}$ and $k_{2}$, respectively.

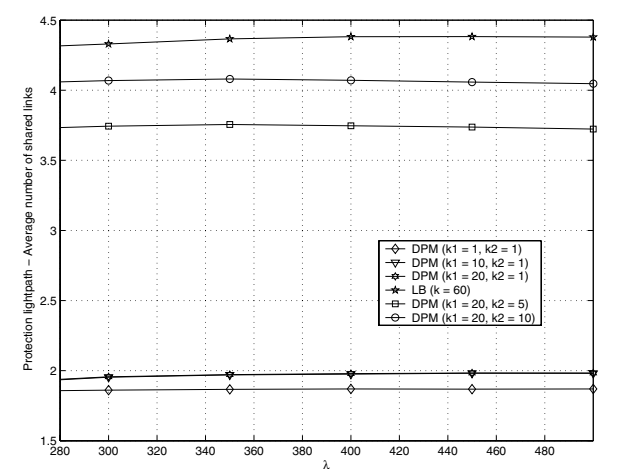

Fig. 6. Average number of shared links per protection path

\section{CONCLUSION}

The Disjoint Path-Pair Matrix (DPM) approach was presented in the paper with the aim to reduce the Routing and Wavelength Assignment (RWA) problem complexity in reliable WDM networks. The DPM approach was shown to be effective in solving the online RWA problem when the shared path protection switching scheme with differentiated reliability is applied to the European network topology. Specifically, the DPM approach was shown to yield blocking probabilities that are comparable to those obtained by the more complex $k$-shortest paths approach. Another advantage of the DPM approach is the contained expected hop length of both the chosen working and protection paths. In addition, the expected hop length difference between the working and protection
TABLE II

Network PeRformance With DifFerent VAlues $k_{1}$ AND $k_{2}$

\begin{tabular}{|c|c|c|c|c|c|c|}
\hline Scheme & $k_{1}$ & $k_{2}$ & $\lambda$ & $B P$ & $H_{W}$ & $H_{P}$ \\
\hline LB & \multicolumn{2}{|c|}{$k=60$} & 300 & $1.7 \cdot 10^{-3}$ & 2.391 & 4.559 \\
\hline DPM & 30 & 5 & 300 & $1.4 \cdot 10^{-3}$ & 2.342 & 3.916 \\
\hline DPM & 20 & 10 & 300 & $1.4 \cdot 10^{-3}$ & 2.317 & 4.246 \\
\hline DPM & 20 & 5 & 300 & $1.4 \cdot 10^{-3}$ & 2.319 & 3.927 \\
\hline DPM & 10 & 10 & 300 & $2.2 \cdot 10^{-3}$ & 2.279 & 4.249 \\
\hline DPM & 10 & 5 & 300 & $2.4 \cdot 10^{-3}$ & 2.282 & 3.942 \\
\hline
\end{tabular}

paths chosen by the DPM approach is less than the expected hop length difference obtained by the commonly used $k$ shortest paths approach.

Given these encouraging results, it is reasonable to expect that the proposed DPM approach yields similar advantages in a number of other complex protection switching problems, including a variety of offline resource provisioning designs [17].

\section{REFERENCES}

[1] I. Chlamtac, A. Ganz, and G. Karni, "Lightpath Communications: An Approach to High Bandwidth Optical WANs," IEEE/ACM Transactions on Communications, vol. 40, no. 7, pp. 1171-1182, July 1992.

[2] "Optical Network Magazine, Special Issue on Protection and Survivability in Optical Network," vol. 2. SPIE, Kluwer, July/August 2001.

[3] G. Mohan, C. S. R. Murthy, and A. Somani, "Efficient Algorithms for Routing Dependable Connections in WDM Optical Networks," IEEE/ACM Transaction on Networking, vol. 9, no. 5, pp. 553-566, October 2001.

[4] H. Zang, J. Jue, and B. Mukherjee, "Review of Routing and Wavelength Assignment Approaches for Wavelenght-Routed Optical WDM Networks," Optical Network Magazine, 2000.

[5] C. Ou, J. Zhang, H. Zang, L. Sahasrabuddhe, and B. Mukherjee, "Online Algorithms for Shared-Path Protection in WDM Mesh Networks," Department of Computer Science, University of California, Davis, Tech. Rep. CSE-2002-6, March 2002.

[6] M. Gondram and M. Minoux, Graph and Algorithms. Wiley Interscience, 1979.

[7] S. D. C. Xin, Y. Ye and C. Qiao, "A Joint Working and Protection Path Selection Approach in WDM Optical Networks," in Proc. IEEE GLOBECOM'01, vol. 4, November 2001, pp. 2165-2168.

[8] J. Y. Yen, "Finding the K Shortest Loopless Paths in a Network," Management Science, vol. 17, pp. 712-716, 1971.

[9] J. Suurballe, "Disjoint Paths in a Network," Networks, vol. 4, pp. 125$145,1974$.

[10] A. Fumagalli, P. Monti, and M. Tacca, "A Differentiated Reliability (DiR) Approach for Dynamic Provisioning in WDM Networks," in Proc. 40th Annual Conference on Communication, Control, and Computing, October 2002.

[11] M. Tacca, "Differentiated Reliability in WDM Networks," Ph.D. dissertation, University of Texas at Dallas, May 2002

[12] K. Gummadi, M. Pradeep, and C. S. R. Murthy, "An Efficient PrimarySegmented Backup Scheme for Dependable Real-Time Communication in Multihop Networks," IEEE/ACM Transaction on Networking, vol. 11, no. 1, pp. 81-94, February 2003.

[13] A. Fumagalli, M. Tacca, F. Unghváry, and A. Faragó, "Shared Path Protection with Differentiated Reliability," in Proc. IEEE ICC 2002, vol. 4, April/May 2002, pp. 2157-2161.

[14] G. N. Rouskas and H. G. Perros, "A Tutorial on Optical Networks," in NETWORKING 2002 Tutorials, ser. Lecture Notes in Computer Science, vol. 2497. Springer, 2002.

[15] D. Dunn, W. Grover, and M. MacGregor, "Comparison of k-Shortest Path and Maximum Flow Routing for Network Facility Restoration," IEEE Journal on Selected Areas in Communications, vol. 12, no. 1, pp. 88-99, January 1994.

[16] P. Ho and H. Mouftah, "An Approach for Enhancing Fixed Alternate Routing in Dynamic Wavelength-Routed WDM Networks," in Proc. IEEE GLOBECOM'02, November 2002, pp. 2792-2797.

[17] R. Ramaswami and K. N. Sivarajan, Optical Networks: a Practical Perspective. Morgan Kaufmann Publishers, Inc., 1998. 